\title{
LncRNA SLCO4A1-AS1 facilitates growth and metastasis of colorectal cancer through $\beta$-catenin-dependent Wnt pathway
}

\author{
Jiangtao Yu', Zhiyang Han ${ }^{1,2}$, Ziquan Sun'², Yue Wang ${ }^{3}$, Ming Zheng ${ }^{4}$ and Chunfang Song ${ }^{1 *}$
}

\begin{abstract}
Background: Emerging evidence has shown long noncoding RNAs (IncRNAs) exert important roles in colorectal cancer (CRC) tumorigenesis. However, most IncRNAs involved in this process remain undefined and the underlying molecular mechanisms mediated by IncRNAs are largely unknown.

Methods: An unbiased screening was used to identify novel IncRNAs involved in CRC according to an onlineavailable data dataset. In situ hybridization (ISH) and qRT-PCR was used to detect IncRNA expression patterns. CCK8, colony formation, fluorescence activated cell sorter (FACS), transwell, xenograft nude mouse model and western blot assays were used to analyze the functions of SLCO4A1-AS1. RNA-pulldown, western blot, RNA fluorescence in situ hybridization (RNA-FISH) and electrophoretic mobility shift assay (EMSA) assays were utilized to explore the molecular mechanism of SLCO4A1-AS1.

Results: LnCRNA SLCO4A1-AS1 was significantly upregulated in CRC tissues and its overexpression was closely related with poor prognosis and tumor metastasis. By knocking down SLCO4A1-AS1, we found that SLCO4A1-AS1 promoted the proliferation, migration, invasion and epithelial-mesenchymal transition (EMT) of CRC cells in vitro, as well as inhibited cell apoptosis. Moreover, SLCO4A1-AS1 dramatically delayed tumor propagation in vivo. Mechanistically, SLCO4A1-AS1 activates Wnt/ $\beta$-catenin signaling. SLCO4A1-AS1 enhanced the stability of $\beta$-catenin by impairing the interaction of $\beta$-catenin with GSK $\beta$ and inhibiting its phosphorylation. Finally, restoration of $\beta$ catenin protein level rescued the proliferation, migration and invasion in SLCO4A1-AS1-depleted CRC cells.
\end{abstract}

Conclusion: SLCO4A1-AS1 serves as an oncogenic role in CRC through activating Wnt/ $\beta$-catenin signaling pathway. And SLCO4A1-AS1 might be a useful biomarker for CRC diagnosis and prognosis.

Keywords: SLCO4A1, AS1, Colorectal cancer, Tumorigenesis, $\beta$-catenin

\section{Background}

As one of the most prevalent cancers, colorectal cancer (CRC) has become the third-leading cause of cancer-related death worldwide every year [1]. Hyperactivation of some signaling pathways, including wnt/ $\beta$-catenin, PI3K/AKT, JAK/STAT signaling pathways and so on, often contributes to the development, progression, metastasis and resistance to chemotherapy of CRC [2-4]. Therefore, understanding the underlying molecular mechanisms that regulate these

\footnotetext{
*Correspondence: cf_song01@163.com

${ }^{1}$ Department of General Surgery, the Sanya Hongsen Hospital of Harbin

Medical University, Fenghuang Road, Sanya 572000, China

Full list of author information is available at the end of the article
}

signaling pathways is urgent and essential for developing efficient target-specific drugs against CRC.

Long noncoding RNAs (lncRNA) are a class of transcripts of longer than 200 nucleotides and possess no protein-coding potential [5-7]. Emerging evidence shows that lncRNAs are critical regulators involved in various biological processes via multiple mechanisms $[8$, 9], such as development, immune regulation and especially tumorigenesis [10-12]. Importantly, accumulating studies have proven that aberrant expression of lncRNAs is closely related to various human cancers [13-15]. For example, IncRNA TUG1 interacts with miR-138-5p to enhance cervical cancer progression by upregulating SIRT1 expression [16]. LncRNA SNHG15 recruits EZH2

(C) The Author(s). 2018 Open Access This article is distributed under the terms of the Creative Commons Attribution 4.0 International License (http://creativecommons.org/licenses/by/4.0/), which permits unrestricted use, distribution, and 
to inhibit P15 and KLF2 expression, and promote pancreatic cancer proliferation [17]. In addition, elevated expression of SNHG6 promotes gastric cancer cell proliferation and EMT, and correlates with poor prognosis. This trend has been observed and proven in diverse tumors including prostate cancer, breast cancer, CRC and so on [18-20]. Recently, many lncRNAs such as LINC01567, HOXB-AS3 and BANCR are reported to be involved in the occurrence and metastasis of CRC [21-23]. However, the underlying molecular mechanism through which lncRNAs modulate CRC development and progression remains largely unknown.

In this study, we found that many lncRNAs were differentially expressed in CRC tissues and normal tissues. Among them, SLCO4A1-AS1 was the most upregulated lncRNA. Evidence from TCGA database also showed that SLCO4A1-AS1 possesses a high frequency of copy number amplification in various cancers, especially in CRC. In addition, the overexpression of SLCO4A1-AS1 is linked to poor prognosis and tumor malignance in CRC. Knockdown of SLCO4A1-AS1 significantly inhibited the proliferation, migration and invasion of CRC cells and induced apoptosis in vitro and in vivo. Mechanistically, SLCO4A1-AS1 remarkably activates Wnt/ $\beta$-catenin signaling pathway. SLCO4A1-AS1 enhanced the stability of $\beta$-catenin by inhibiting GSK $\beta$-mediated phosphorylation. Altogether, we demonstrated that SLCO4A1-AS1 is an oncogene in CRC by activation of the $\beta$-catenin signaling.

\section{Methods}

\section{Human samples}

Human colorectal samples were collected from the First Affiliated Hospital of Harbin Medical University. And the clinicopathological features were listed in Table 1. These samples were divided into SLCO4A1-AS1 high or low group (the median value of SLCO4A1-AS1 expression as the cutoff), followed by survival rate analysis. Informed consent allowing use of these samples was obtained from each patient. Samples were processed according to the standard procedures with appropriate ethical approval by the Ethics Committee of the First Affiliated Hospital of Harbin Medical University.

\section{Cell lines and cell culture}

Human normal colorectal mucosa cell FHC and CRC cell lines (HCT116, HCT8, HT29, SW480, LOVO and SW620) were purchased from the American Type Culture Collection (ATCRC) and maintained according to the standard procedures.

\section{Cell transfection}

shRNAs (shSLCO4A1-AS1: 5'-GCCTGAGCTTGTTC ACAAA-3') were designed using Clontech RNAi Target
Table 1 Association between SLCO4A1-AS1 expression and clinicopathological characteristics in 50 patients with CRC

\begin{tabular}{|c|c|c|c|c|}
\hline Characteristics & $\begin{array}{l}\text { SLCO4A1-AS1 } \\
\text { Low }\end{array}$ & $\mathrm{High}^{\mathrm{a}}$ & Chi-square & $P$ value \\
\hline All cases & 21 & 29 & & \\
\hline Age (year) & & & 1.643 & 0.200 \\
\hline$<60$ & 16 & 26 & & \\
\hline$\geq 60$ & 5 & 3 & & \\
\hline Gender & & & 0.006 & 0.939 \\
\hline Male & 15 & 21 & & \\
\hline Female & 6 & 8 & & \\
\hline Size $(\mathrm{cm})$ & & & 4.711 & 0.030 \\
\hline$<3$ & 13 & 9 & & \\
\hline$\geq 3$ & 8 & 20 & & \\
\hline Lymph node metastasis & & & 4.217 & 0.040 \\
\hline No & 11 & 7 & & \\
\hline Yes & 10 & 22 & & \\
\hline TNM & & & 4.433 & 0.035 \\
\hline$|/| \mid$ & 12 & 8 & & \\
\hline III/IV & 9 & 21 & & \\
\hline
\end{tabular}

${ }^{a}$ The median expression level was chosen for cutoff For analysis of correlation between SLCO4A1-AS1 levels and clinical features, Pearson's chi-square tests were used. Results were considered statistically significant at $P<0.05$

Sequence Selector and constructed into the pSiCoR plasmid according to the instructions. Two hundred ninety-three $\mathrm{T}$ cells transfected with $\mathrm{pSiCoR}$ as well as VSVG, RRE and RSV-REV were used to generate virus. HCT116 and SW480 cells were infected with virus supernatants. Stable cell lines were isolated by GFP sorting.

\section{Antibodies}

Antibodies for Vimentin (1:2000; \#5741, Cell Signaling Technology), E-cadherin (1:1000; \#14472, Cell Signaling Technology), AXIN2 (1:2000; \#5863, Cell Signaling Technology), MYC (1:5000; \#13987, Cell Signaling Technology), $\beta$-catenin (1:1000; \#8480, Cell Signaling Technology), phospho- $\beta$-catenin (T41, S45) (1:1000; \#9565, Cell Signaling Technology), GSK3 $\beta$ (1:1000; \#12456, Cell Signaling Technology) and GAPDH (1:5000; \#5014, Cell Signaling Technology) were bought from Cell Signaling Technology. $\alpha$-catenin (1:2000; \#610194, BD) was from BD Transduction Laboratories. Fibronectin (1:1000; SAB4500974, Sigma) and LGR5 (1:1000; SAB2700211, Sigma) were from Sigma.

\section{Apoptosis analysis}

Cell apoptosis were analyzed by flow cytometry (FACScan; BD Biosciences) using CellQuest software (BD Biosciences). 


\section{Ubiquitination assay}

This assay was carried out as described before [24]. In brief, HA-ubiquitin vector were transfected into CRC cells. Forty-eight $\mathrm{h}$ after transfection, cells were treated with MG132 $(10 \mu \mathrm{M} / \mathrm{L})$ for $10 \mathrm{~h}$. Then cell lysates were pulled down using anti- $\beta$-catenin or IgG. Eluates were separated by SDS-PAGE and immunoblotted with anti-K48-Ub.

\section{Tumorigenesis and metastasis assays in vivo}

Animal experiments were $\mathrm{s}$ approved by the Medical Experimental Animal Care Commission at the First Affiliated Hospital of Harbin Medical University. To assess the effect in vivo of SLCO4A1-AS1 on tumorigenesis, shSLCO4A1-AS1 or control SW480 cells $\left(2 \times 10^{6}\right.$ cells per mouse) were injected into the flanks of 5 -week-old athymic nude BALB/c mice were manipulated nude recipients subcutaneously. Tumor volumes weights were determined at indicative time points. To evaluate the effects of SLCO4A1-AS1 on tumor metastasis in vivo, shSLCO4A1-AS1 or control SW480 cells $\left(4 \times 10^{6}\right.$ cells per mouse) were injected into the spleen subcapsular of each BALB/c nude mice. Six weeks after injection, metastatic nodules in livers were counted under a dissecting microscope.

\section{Cell proliferation assays}

CCK- 8 and colony formation assays were used for evaluation of cell proliferation ability. For CCK- 8 assay, cells were seeded into a 96-well plate. At indicative time points, $10 \mu \mathrm{l}$ CCK- 8 solution was added into each well and incubated for $2 \mathrm{~h}$ at $37{ }^{\circ} \mathrm{C}$. Then, the absorbance at $450 \mathrm{~nm}$ was determined. For the colony formation assay, $2 \times 10^{3}$ cells per well were seeded in a six-well plate and cultured for 2 weeks. Colonies were fixed with methanol and stained with $0.1 \%$ crystal violet $(1 \mathrm{mg} / \mathrm{ml})$.

\section{In vitro migration and invasion assay}

For migration assay, $2 \times 10^{4}$ cells were seeded in the top chamber $(8-\mu \mathrm{m}$ pore; BD Biosciences) with serum-free medium and the lower chamber was added with $10 \%$ fetal bovine serum medium. Cells were incubated for $24 \mathrm{~h}$ and then non-migrated cells were removed and the migrated cells on the lower side were fixed, stained with crystal violet and photographed with an IX71 inverted microscope (Olympus Corp., Tokyo, Japan). For invasion assay, $8 \times 10^{4}$ cells were placed in the upper chamber coated with $100 \mu \mathrm{l}$ Matrigel (BD Biosciences, MA). And other steps were the same as migration assay.

\section{Real-time quantitative PCR}

TRIzol solution was used to extract total RNAs from sample tissues or cell lines according to the manufacturer's protocol. The M-MLV reverse transcriptase (Promega) was used for cDNA synthesis. qRT-PCR was performed as previously described [25]. Gene expression was normalized to U6 or GAPDH and calculated according to the $2^{-\Delta \Delta C T}$ method. Specific primer sequences were available if requested.

\section{In situ hybridization (ISH)}

ISH were conducted as previously described [26]. The probe sequences for SLCO4A1-AS1 were as follows: 5'-GAAGCTAGATGCRCAGCTAAT-3' and 5' -TTGC GTTCATCGGAACRCAGG-3'.

\section{RNA immunoprecipitation (RIP)}

Cells were lysed with RIP buffer $(20 \mathrm{mM}$ Tris $\mathrm{pH} 7.5$, $150 \mathrm{mM} \mathrm{NaCl}, 1 \mathrm{mM} \mathrm{MgCl} 2,0.1 \%$ NP40, 5\% glycerol and $0.5 \mathrm{mM} \mathrm{DTT}$ ) supplemented with RNase inhibitor, followed by addition of specific antibody. RNA-protein complexes were enriched by Protein A/G beads. Then precipitated RNAs were eluted and used for cDNA synthesis.

\section{RNA pulldown}

Biotin-labeled RNAs were obtained by the MaxiScript T7 kit (Ambion) with biotinylated CTP. Biotin-labeled RNAs in refolding buffer $(10 \mathrm{mM}$ Tris $\mathrm{pH} 7.5,0.1 \mathrm{M} \mathrm{KCl}$ and $10 \mathrm{mM} \mathrm{MgCl} 2$ ) were added into cell lysates and incubated for $4 \mathrm{~h}$ at $4{ }^{\circ} \mathrm{C}$, followed by addition of beads. After washed 4 times, beads were boiled and precipitated proteins were checked with western blot.

\section{Statistical analysis}

SPSS version 20.0 software (SPSS Inc., Chicago, IL, USA) was used for statistical analysis. Data was expressed as mean \pm SD. Survival curves were calculated using the Kaplan-Meier curve followed by log-rank test. One-way ANOVA analysis or two-tailed Student's t-tests were performed for $p$-value analysis, as appropriate. $P<0.05$ was considered statistically significant.

\section{Results}

\section{SLCO4A1-AS1 is overexpressed in human CRC}

To identify CRC-related lncRNAs, we analyzed an online non-coding RNA profiling according to Li's cohort (GSE104836) consisting of 10 pairs of tumor and adjacent normal tissues. Among all differentially expressed lncRNAs between tumor and normal tissues, SLCO4A1-AS1 was the most upregulated in tumor samples (Fig. 1a). Previous study showed that copy number amplification (CNA) is linked to upregulation of oncogene expression and subsequent tumorigenesis [27]. According to TCGA database, we found that the frequency of CNA of SLCO4A1-AS1 was very high in various cancers, including about $9 \%$ in CRC (Fig. 1b). To validate it, we collected 50 CRC samples and detected the copy number of SLCO4A1-AS1 by qPCR. 


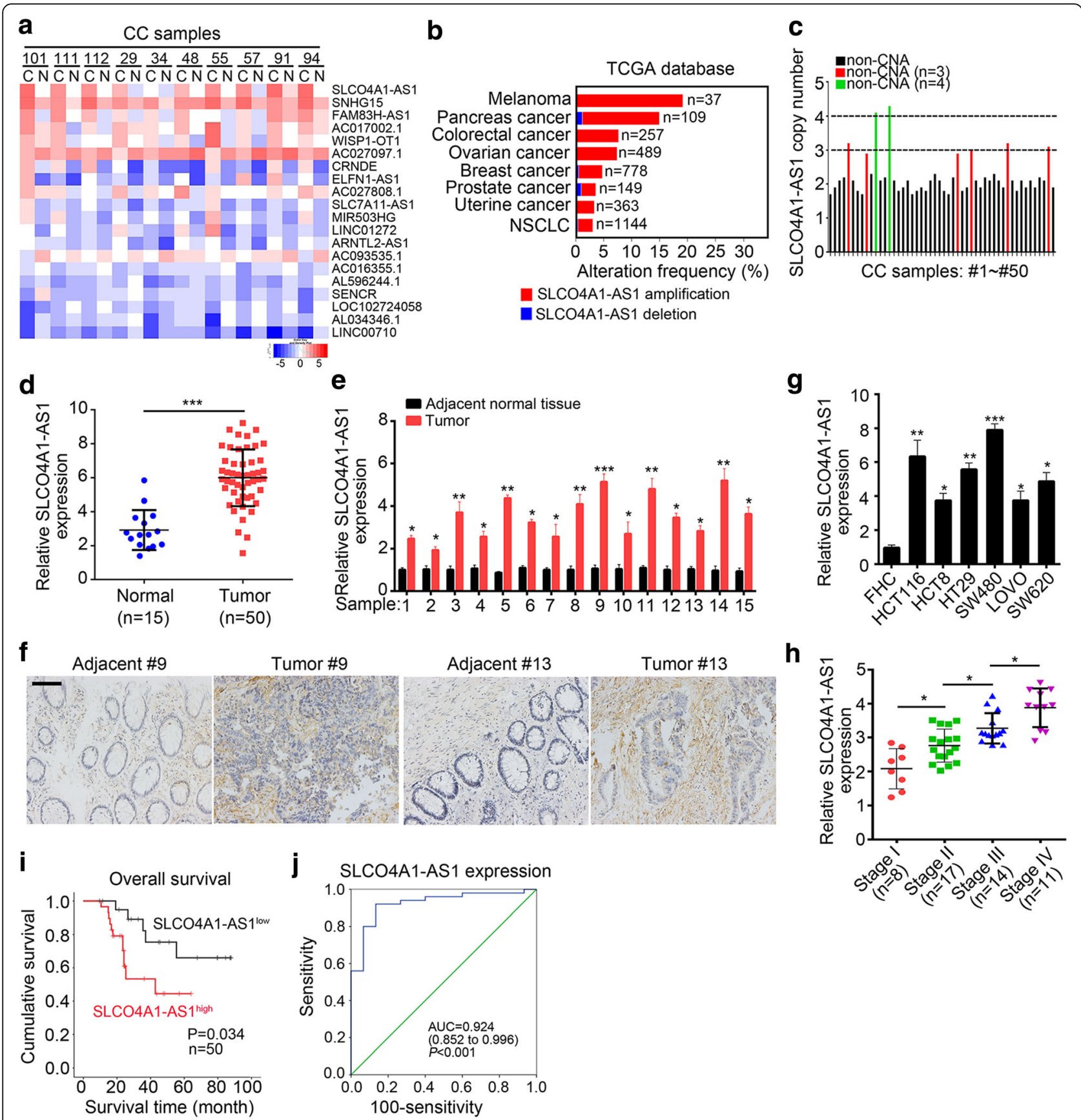

Fig. 1 SLCO4A1-AS1 is overexpressed in human CRC. a Heatmap of differentially expressed long noncoding RNAs in CRC tissues and adjacent non-tumor tissues according to an online microarray dataset (GSE104836). b The copy number amplifications (CAN) of SLCO4A1-AS1 were determined according to TCGA database. $\mathbf{c}$ The CNA of SLCO4A1-AS1 was measured by qRT-PCR in 50 CRC samples. DNAs were extracted from samples and specific primers targeting SLCO4A1-AS1 DNA were used. ACTB DNA was used for loading control. d SLCO4A1-AS1 expression was determined by qRT-PCR in CRC samples $(n=50)$ and adjacent normal tissues $(n=15)$. e SLCO4A1-AS1 expression was examined by qRT-PCR in 15 pairs of CRC samples and adjacent normal tissues. $\mathbf{f}$ The expression of SLCO4A1-AS1 was determined by in situ hybridization in pairs of CRC samples and adjacent normal tissues (\#9 and \#13). Scale bar, $50 \mu \mathrm{m}$. $\mathbf{g}$ The expression levels of SLCO4A1-AS1 were determined by qRT-PCR in human normal colorectal mucosa cell FHC and CRC cell lines (HCT116, HCT8, HT29, SW480, LOVO and SW620). h 50 CRC samples were divided into four groups based on clinical stages (TNMS). And the expression of SLCO4A1-AS1 was measured in each group by qRT-PCR. $\mathbf{i}$ The effect of SLCO4A1-AS1 expression on clinical prognosis was determined by Kaplan-Meier survival analysis. Median value was the cutoff threshold of Kaplan-Meier survival analysis. $\mathbf{j}$ Prediction of CRC based on SLCO4A1-AS1 expression was analyzed by ROC curve using adjacent normal tissues as control. ${ }^{*} p<0.05,{ }^{* *} p<0.01$ and ${ }^{* * *} p<0.01$ 
We found that 8 samples had copy number amplification including 6 3-copy and 2 4-copy samples (Fig. 1c), which implied that SLCO4A1-AS1 may be involved in CRC development. To further validate it, we measured the expression of SLCO4A1-AS1 in CRC samples and adjacent normal tissues. We found that SLCO4A1-AS1 was significantly upregulated in CRC tissues compared with paired normal tissues (Fig. 1d and e). ISH assay also showed that the expression of SLCO4A1-AS1 was higher in CRC samples (Fig. 1f). Consistently, the expression of SLCO4A1-AS1 was higher in CRC cell lines (HCT116, HCT8, HT29, SW480, LOVO and SW620) than that in human normal colorectal mucosa cell FHC (Fig. 1g). To further determine the relationship between SLCO4A1-AS1 expression and tumor malignance, we checked the expression of SLCO4A1-AS1 in different stages of CRC samples and found that SLCO4A1-AS1 expression is positively correlated with clinical grade (Fig. 1h). Furthermore, by Kaplan-Meier survival analysis, we found that CRC patients with higher expression of SLCO4A1-AS1 showed poorer prognosis (Fig. 1i). In addition, receiver operating characteristic (ROC) curve was performed to evaluate the sensitivity and specificity of SLCO4A1-AS1 expression in predicting CRC tissues from normal tissues. The area under curve (AUC) was 0.924, which indicated SLCO4A1-AS1 might be a good predictor in CRC (Fig. 1j). Collectively, above results implied that SLCO4A1-AS1 was significantly upregulated and might serve as a biomarker for prognosis in CRC.

\section{SLCO4A1-AS1 knockdown inhibits cell proliferation, migration and invasion in CRC}

To investigate the role of SLCO4A1-AS1 in CRC, we knocked down SLCO4A1-AS1 with two independent siRNAs in HCT116 and SW480 cells (Fig. 2a). CCK-8 assays showed that SLCO4A1-AS1 knockdown led to growth retardation of HCT116 and SW480 cells (Fig. 2b). Colony formation assay similarly indicated that SLCO4A1AS1-depleted CRC cells formed fewer colonies than the controls (Fig. 2c). Predictably, SLCO4A1-AS1 significantly inhibited the percent of CRC cells in S phase (Fig. 2d). In addition, Transwell assays showed that SLCO4A1-AS1 silencing decreased CRC cell migration (Fig. 2e) and invasion (Fig. 2f). Besides, we performed western blot to evaluate whether SLCO4A1-AS1 regulates pithelial-mesenchymal transition (EMT) in CRC cells. Results demonstrated that knockdown of SLCO4A1-AS1 upregulated the expression of epithelial markers such as E-cadherin, $\alpha$-catenin while decreased the expression of mesenchymal markers such as Vimentin and Fibronectin (Fig. 2g). Finally, we assessed the effect of SLCO4A1-AS1 on cell apoptosis. By staining with Annexin V/PI, we found that SLCO4A1-AS1 knockdown dramatically promoted the apoptosis (Fig. 2h). Taken together, our data demonstrated that SLCO4A1-AS1 knockdown could inhibit CRC proliferation and invasion in vitro.

\section{SLCO4A1-AS1 activates Wnt/ $\beta$-catenin signaling in CRC}

To further determine SLCO4A1-AS1-mediated molecular mechanism, we performed analysis in bioinformatics according to the Li's cohort (GSE104836). We divided the 10 CRC samples in Li's cohort into two groups based on SLCO4A1-AS1 expression (median value as the cutoff). Gene Set Enrichment Analysis (GSEA) showed that SLCO4A1-AS1 highly expression was positively related to cell division and G1/G2 phase transition (Fig. 3a), which supported our above results that SLCO4A1-AS1 promoted CRC cell proliferation. Some signaling pathways such as Wnt/ $\mathrm{W}$-catenin signal and $\mathrm{NOTCH}$ signal were reported to be involved in human cancers [28, 29]. We analyzed the relationship of SLCO4A1-AS1 with cancer-related signaling pathways and found that SLCO4A1-AS1 expression was positively correlated with Wnt $/ \beta$-catenin signaling pathway (Fig. $3 \mathrm{~b}$ ). As shown, many target genes of $\mathrm{Wnt} / \beta$-catenin signaling was downregulated in SLCO4A1-AS1 ${ }^{\text {low }}$ CRC samples compared with SLCO4A1-AS1 ${ }^{\text {high }}$ samples (Fig. 3c). Similarly, SLCO4A1-AS1 knockdown significantly decreased the mRNA and protein levels of these target genes in HCT116 and SW480 cells (Fig. 3d-f). What's more, the expression levels of AXIN2, LGR5, SOX4 and MYC were positively correlated with that of SLCO4A1-AS1 in the 50 CRC sample tissues (Fig. 3j), which indicated that SLCO4A1-AS1 regulates Wnt/ $\beta$-catenin signaling in CRC.

\section{SLCO4A1-AS1 interacts with $\beta$-catenin}

We above showed that SLCO4A1-AS1 activated Wnt/ $\beta$-catenin signaling in CRC. To determine how SLCO4A1-AS1 activates Wnt/ $\beta$-catenin signaling, we performed RNA pulldown assays, followed by silver staining and mass spectrum (MS) identification. We identified $\beta$-catenin as an interactive protein of SLCO4A1-AS1 (Fig. 4a). To verify it, we performed pulldown assay and found that biotin-labeled SLCO4A1-AS1 precipitated endogenous $\beta$-catenin in HCT116 and SW480 cells (Fig. 4b). Furthermore, $\beta$-catenin antibody also enriched endogenous SLCO4A1-AS1 in HCT116, SW480 and CRC sample cells (Fig. 4c and d). In addition, SLCO4A1-AS1 co-localized with $\beta$-catenin in CRC sample cells (Fig. 4e). To further determine the essential region in SLCO4A1AS1 for the interaction with $\beta$-catenin, we conducted domain mapping. We found that SLCO4A1-AS1 (nt 900 1200) directly bound to $\beta$-catenin (Fig. 4f). Moreover, deletion of this region (nt 900 1200) abrogated this interaction between SLCO4A1-AS1 and $\beta$-catenin (Fig. 4g). Furthermore, we performed RNA electrophoretic mobility shift assay (RNA-EMSA) with biotin-labeled 


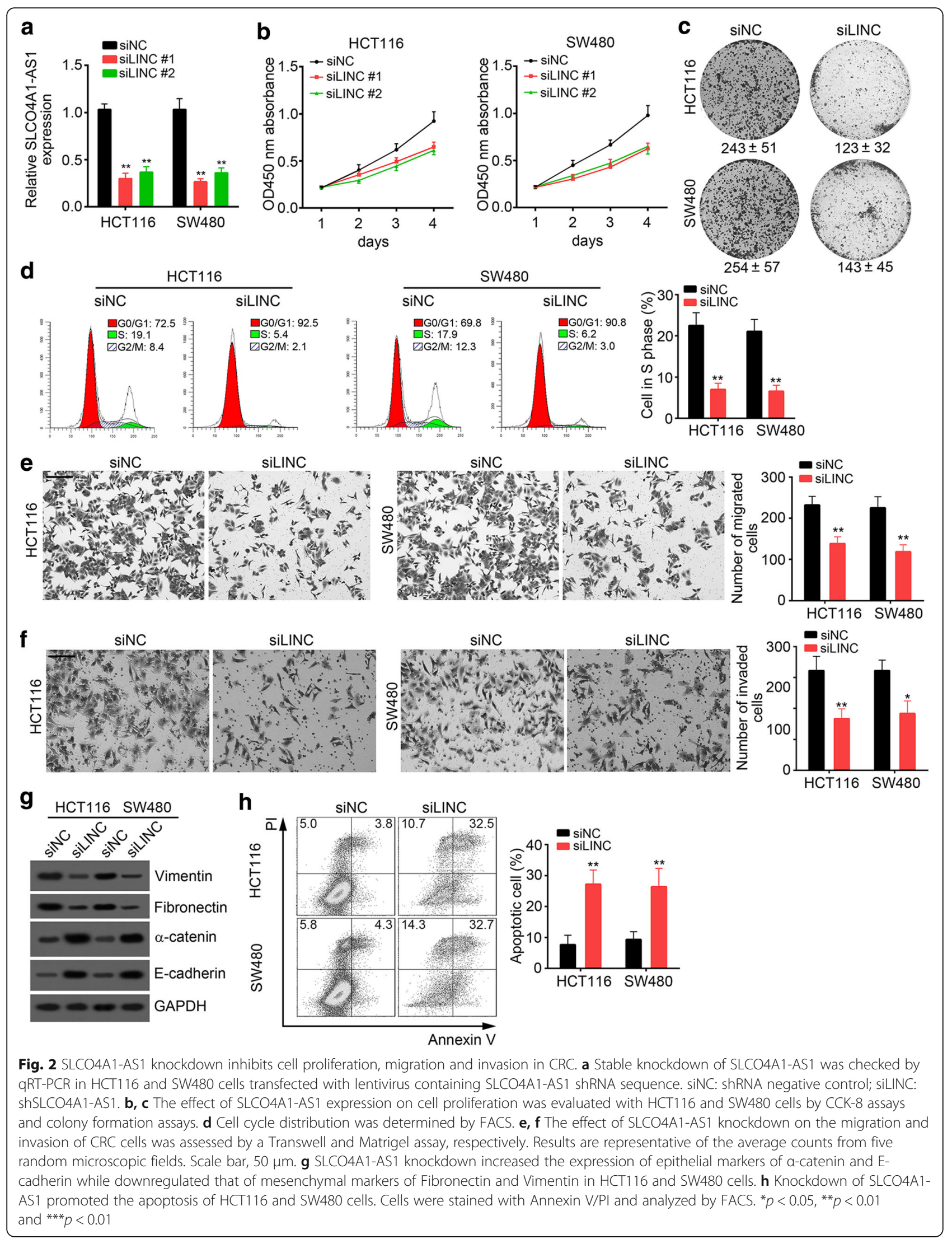




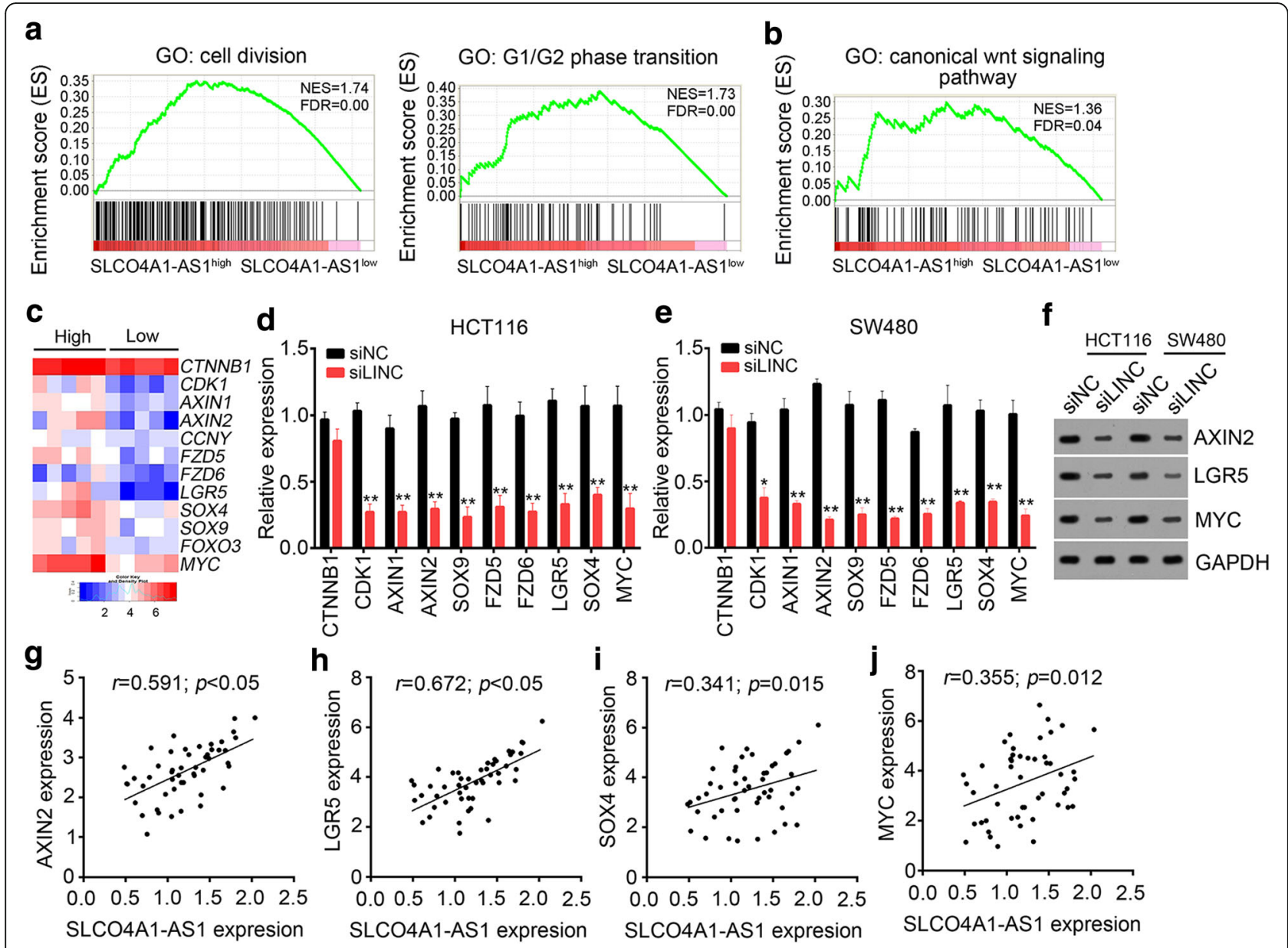

Fig. 3 SLCO4A1-AS1 activates Wnt/B-catenin signaling in CRC. a Gene Set Enrichment Analysis (GSEA) showed that SLCO4A1-AS1 highly expression was positively related to cell division and G1/G2 phase transition. Samples in Li's cohort (GSE104836) were divided into 2 groups based on expression of SLCO4A1-AS1. NES, normalized enrichment score. b GSEA showed that SLCO4A1-AS1 expression was positively correlated with canonical Wnt signaling according to Li's cohort (GSE104836). c Expression heatmap of the target genes of Wnt/ 3 -catenin signaling showed that more activation of Wnt/ $\beta$-catenin signaling appeared in SLCO4A1-AS1 ${ }^{\text {high }}$ group. $\mathbf{d}$, e Real-time PCR confirmed that SLCO4A1-AS1 knockdown significantly downregulated Wnt/ $\beta$-catenin signaling in HCT116 and SW480 cells. $\mathbf{f}$ The protein levels of AXIN2, LGR5 and MYC in HCT116 and SW480 cells after SLCO4A1-AS1 silence were measured by western blot (WB). $\mathbf{g}$-j The mRNA levels of AXIN2, LGR5, SOX4 and MYC were positively correlated with the expression of SLCO4A1-AS1 in the 50 CRC samples. qRT-PCR was performed and GAPDH acted as loading control. ${ }^{*} p<0.05$ and ${ }^{* *} p<0.01$

probe (nt 900 1200) and demonstrated their direct association (Fig. 4h).

\section{SLCO4A1-AS1 increased the stability of $\beta$-catenin by inhibiting its phosphorylation}

We have confirmed the interaction between SLCO4A1AS1 and $\beta$-catenin. Then we performed western blot and found that SLCO4A1-AS1 knockdown significantly decreased the protein level of $\beta$-catenin in HCT116 and SW480 cells (Fig. 5a). On the contrary, overexpression of full-lengthen or nt 900 1200 dramatically upregulated the protein level of $\beta$-catenin in HCT116 and SW480 cells (Fig. 5b). Additionally, we validated the elevated $\beta$-catenin ubiquitination signals using $\beta$-catenin immunoprecipitates from SLCO4A1-AS1-depleted HCT116 cells through
(Fig. 5c) and consequently decreased $\beta$-catenin stability (Fig. 5d). A previous study showed that $\beta$-catenin phosphorylation by GSK $\beta$ promotes its ubiquitinationmediated degradation [30]. We then assessed the effect of SLCO4A1-AS1 on $\beta$-catenin phosphorylation and found that SLCO4A1-AS1 knockdown significantly increased $\beta$-catenin phosphorylation in HCT116 and SW480 cells (Fig. 5e). Moreover, SLCO4A1-AS1-overexpressed CRC sample tissues showed lower $\beta$-catenin phosphorylation (Fig. 5f). Besides, we found that SLCO4A1-AS1 knockdown enhanced the interaction between $\beta$-catenin and GSK $\beta$ in HCT116 and SW480 cells (Fig. 5g) while overexpressing SLCO4A1-AS1 abrogated their interaction (Fig. 5h). To further determine whether SLCO4A1-AS1 activated Wnt/ $\beta$-catenin signaling by enhancing the 


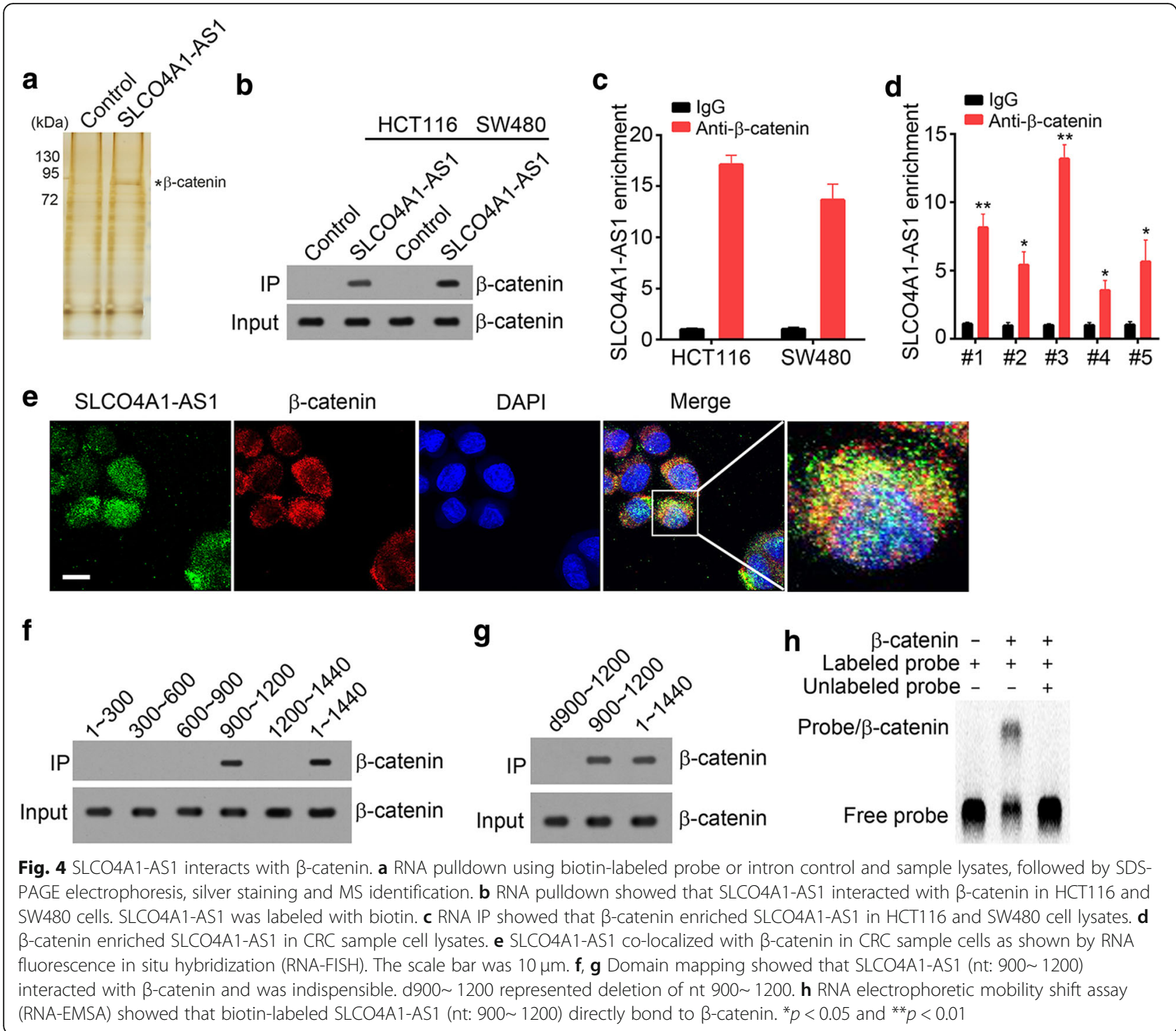

stability of $\beta$-catenin, we restored the protein levels of $\beta$-catenin in HCT116 and SW480 cells (Fig. 5i). By qRT-PCR, we found that restoration of $\beta$-catenin rescued the activation of Wnt/ $\beta$-catenin signaling in HCT116 and SW480 cells (Fig. 5j). Summarily, our results indicated that SLCO4A1-AS1 stabilized $\beta$-catenin by preventing the association between $\beta$-catenin and GSK $\beta$, and consequently activated Wnt/ $\beta$-catenin signaling in CRC.

SLCO4A1-AS1 promotes CRC proliferation, migration and invasion by activating wnt/ $\beta$-catenin signaling in vitro and in vivo

Whether the SLCO4A1-AS1-mediated augment of CRC cell growth and metastasis relied on activation of $\mathrm{Wnt} / \beta$-catenin signaling was assessed in SLCO4A1-AS1-silenced HCT116 and SW480 cells transfected with $\beta$-catenin-overexpressing plasmid or empty control. Results showed that decreased proliferation, colony formation, migration and invasion potentials of SLCO4A1-AS1-silenced cells were rescued by ectopic expression of $\beta$-catenin in HCT116 and SW480 cells (Fig. 6a-d). What's more, SLCO4A1-AS1 knockdown delayed tumor growth in vivo while overexpression of $\beta$-catenin in the meantime reversed it (Fig. $6 \mathrm{e}$ and $\mathrm{f}$ ). Then we measured the activation of $\mathrm{Wnt} / \beta$-catenin signaling in formed tumor tissues. As shown, the Wnt/ $\beta$-catenin signaling was also downregulated in vivo after SLCO4A1-AS1 depletion (Fig. 6g). Finally, we evaluated the effect of SLCO4A1-AS1 on tumor metastasis in vivo, and found that SLCO4A1-AS1 knockdown severely reduced the metastatic nodules in the liver while $\beta$-catenin overexpression reversed this trend (Fig. $6 \mathrm{~h}$ and i). Taken together, above data suggested that SLCO4A1-AS1 exerted functions dependent on activation of $\mathrm{Wnt} / \beta$-catenin signaling in CRC. 

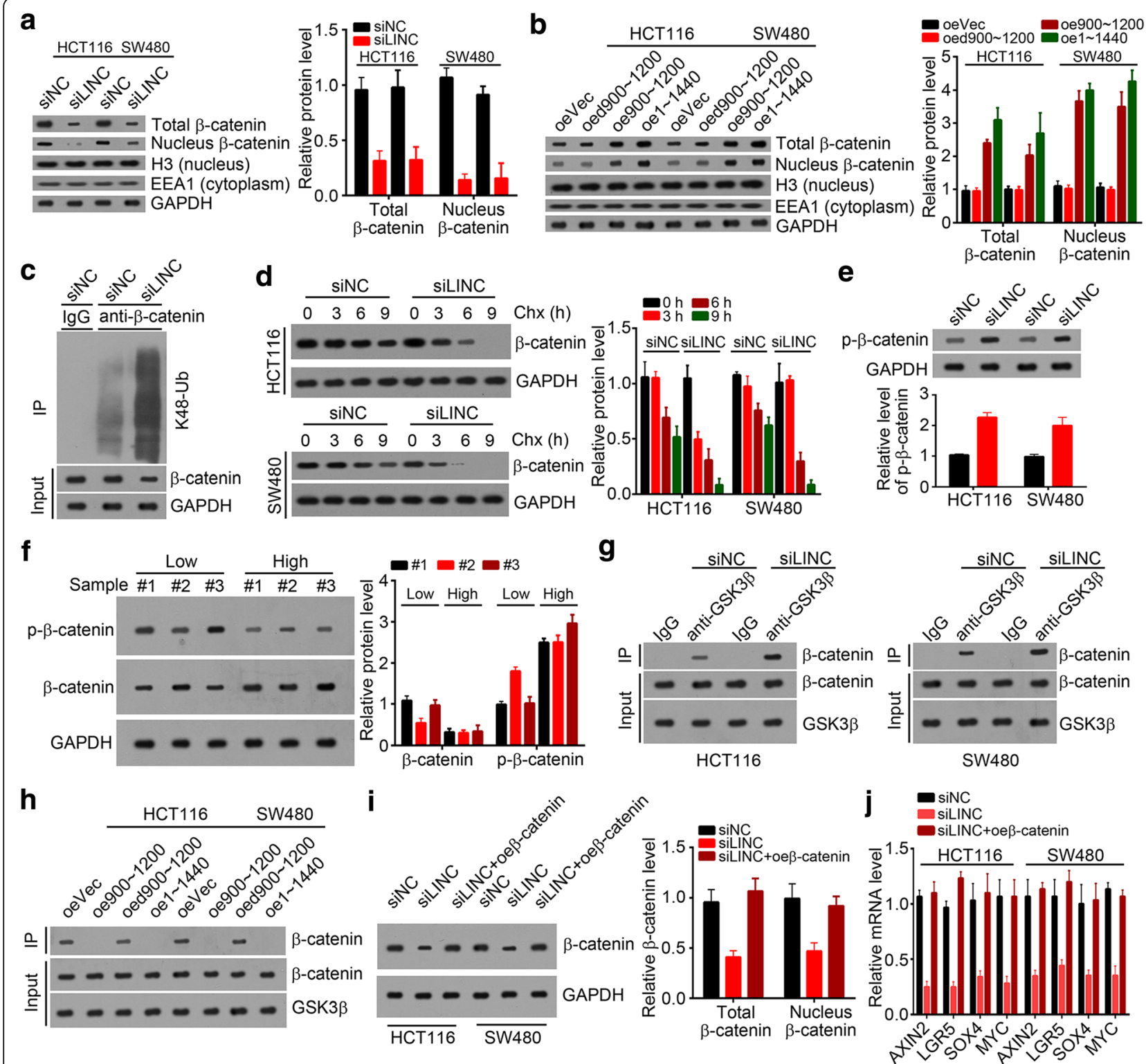

Fig. 5 SLCO4A1-AS1 increased the stability of $\beta$-catenin by inhibiting its phosphorylation. a WB analysis showed that siRNA-induced SLCO4A1AS1 knockdown decreased the protein level of $\beta$-catenin in HCT116 and SW480 cells. H3, nuclear marker; EEA1, cytoplasmic marker. b Overexpression of SLCO4A1-AS1 (full-length or nt 900 1200) promoted the protein level of $\beta$-catenin in HCT116 and SW480 cells. H3, nuclear marker; EEA1, cytoplasmic marker. c Knockdown of SLCO4A1-AS1 enhanced the ubiquitination of $\beta$-catenin in HCT116 cells. d SLCO4A1-AS1 knockdown accelerated the degradation of $\beta$-catenin in HCT116 and SW480 cells. Chx, cycloheximide. e SLCO4A1-AS1 knockdown promoted the phosphorylation of $\beta$-catenin in HCT116 and SW480 cells as shown by western blotting. $\mathbf{f}$ WB analysis showed that the protein levels of $\beta$-catenin were higher in SLCO4A1-AS1 ${ }^{\text {high }}$ CRC samples while the phosphorylation of $\beta$-catenin was lower. $\mathbf{g}$ SLCO4A1-AS1 knockdown enhanced the interaction between $\beta$-catenin and GSK3 $\beta$ in HCT116 and SW480 cells. $\mathbf{h}$ WB analysis indicated that overexpression of SLCO4A1-AS1 (full-length or nt 900 1200) abrogated the interaction between $\beta$-catenin and GSK3 $\beta$ in HCT116 and SW480 cells. $\mathbf{i}$, $\mathbf{j}$ Restoration of the protein levels of $\beta$ catenin by ectopic expression of $\beta$-catenin (i) rescued SLCO4A1-AS1 knockdown-induced inactivation of wnt/ $\beta$-catenin signaling in HCT116 and sW480 cells (j)

\section{Discussion}

In recent years, great efforts have been made to search cancer-related lncRNAs and determine their molecular mechanisms in tumor development and progression [31]. Here we identified the physiological functions of an uncharacterized lncRNA SLCO4A1-AS1 and determined its molecular mechanism. SLCO4A1-AS1 was highly expressed in CRC tissues and may act as a biomarker for CRC diagnosis. Notably, we detected an unbelievable high AUC, which might be due to the limited size of CRC samples. SLCO4A1-AS1 was found to promote the proliferation and invasion of CRC cells, indicating that it 


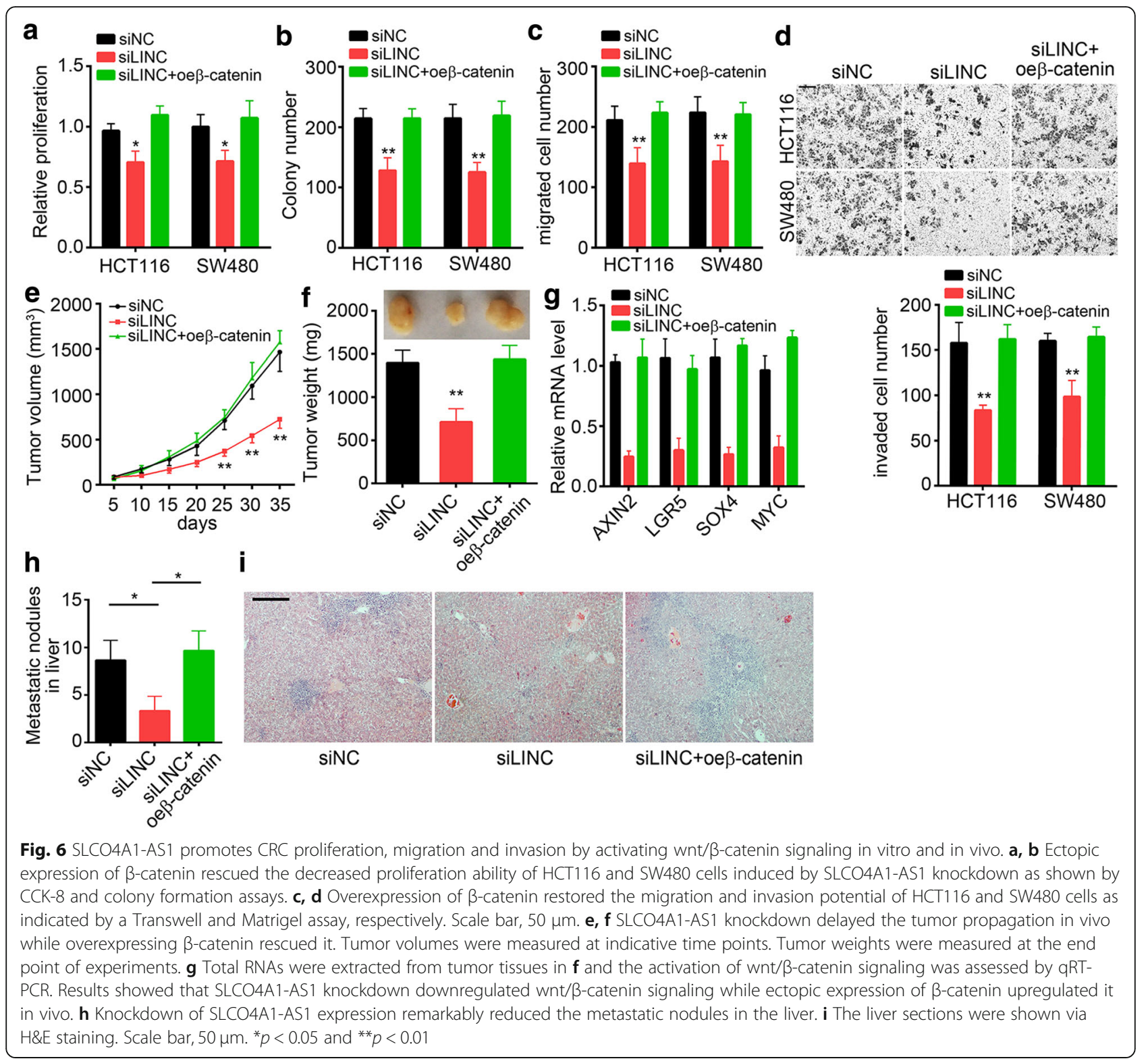

may be implicated in the process of tumorigenesis. Moreover, SLCO4A1-AS1 knockdown induced CRC cell apoptosis, which implied that SLCO4A1-AS1 may be important for the functional maintenance of normal cancer cells.

Further analysis showed that the activation of Wnt/ $\beta$-catenin signaling was affected by SLCO4A1-AS1. As one of the most essential intracellular signaling pathways, $\mathrm{Wnt} / \mathrm{\beta}$-catenin signaling mediates diverse cellular processes, including embryonic development, cell proliferation, differentiation, migration, survival and so on [32-34]. Hyperactivation of the $\mathrm{Wnt} / \beta$-catenin signaling often leads to various cancers such as liver cancer and CRC [35-37]. For instance, CRCAT-1-mediated activation of Wnt signaling pathway promotes cell proliferation and inhibits apoptosis in cervical cancer cells [38]. Additionally, activation of $\mathrm{Wnt} / \beta$-catenin signaling by TGF $\beta$ promotes CRC development [39]. In our study, we found that SLCO4A1-AS1 knockdown severely decreased the protein level of $\beta$-catenin but not mRNA level by the mechanism that SLCO4A1-AS1 inhibited the phosphorylation and consequently ubiquityla tion-mediated degradation of $\beta$-catenin. Through interacting with $\beta$-catenin, SLCO4A1-AS1 impaired the binding of GSK3 $\beta$ to $\beta$-catenin and inhibited $\beta$-catenin phosphorylation by GSK3 $\beta$. Emerging evidence shows that lncRNAs can exert functions by regulation in trans [40]. IncRNAs may associate with proteins to regulate their stability, activity or other properties [11, 41, 42]. Based on above 
evidence, we proposed that SLCO4A1-AS1 may bind to $\beta$-catenin and then shield the interactive domain of $\beta$-catenin with GSK3 $\beta$.

$\beta$-catenin level plays a pivot role in the canonical Wnt pathway [43]. Increase of $\beta$-catenin protein level may lead to abnormal cell proliferation and human diseases [44]. The regulation of $\beta$-catenin protein level is complicated and delicate. Phosphorylation and ubiquitylation of $\beta$-catenin are all reported to participate in the regulation of $\beta$-catenin stability [45]. For example, Liu et al. demonstrated that phosphorylation of $\beta$-catenin by CKI $\alpha$ in vivo is indispensible for subsequent phosphorylation of $\beta$-catenin by GSK3 $\beta$, which finally leads to degradation of $\beta$-catenin [45]. Besides, other studies showed that phosphorylated $\beta$-catenin is ubiquitylated by $\mathrm{E} 3$ ubiquitin ligase $\beta-\operatorname{TrCP}$ and then degraded by the ubiquitin-proteasome pathway [46, 47]. Abrogation of $\beta$-catenin degradation promotes the accumulation of $\beta$-catenin in cells and induces tumor occurrence. For instance, inactivating mutation of APC, a pivot subunit of the degradation complex of $\beta$-catenin, gave rise to spontaneous CRC in mice [48]. So far, the regulatory mechanism of $\beta$-catenin turnover is not fully understood. Our study revealed that SLCO4A1-AS1 regulated the stability of $\beta$-catenin by weakening the association between $\beta$-catenin and GSK3 $\beta$.

Continuous mutations of genes are popularly considered as a cause of tumors [49]. Gene copy number alterations or mutations are the common aberrances in cancers, and some studies have demonstrated the relevance between gene copy-number alterations and tumor formation and progression [50]. Previous study shows that DNA copy-number gain was observed on chromosome $20 \mathrm{q}$ in primary colorectal tumor [51]. Notably, SLCO4A1-AS1 is also located on chromosome 20q. Moreover, SLCO4A1-AS1 is really substantially amplified in CRC according to TCGA database and our experiment (Fig. 1b and c). However, how copy-number amplifications on chromosome 20q affect the expression and functions of SLCO4A1-AS1 in CRC remains further investigation.

\section{Conclusion}

In summary, we found that IncRNA SLCO4A1-AS1 was highly expressed in CRC tissues. Upregulated SLCO4A1AS1 promoted CRC progression through inhibiting the degradation of $\beta$-catenin by attenuating the interaction between $\beta$-catenin and GSK3 $\beta$. This study revealed the vital significance of SLCO4A1-AS1 in CRC development.

\section{Abbreviations \\ CRC: colorectal cancer; EMSA: Electrophoretic mobility shift assay; IncRNA: long noncoding RNA; RNA-FISH: RNA fluorescence in situ} hybridization

Acknowledgements

The authors thank all patients involved in this study.

\section{Funding}

This work was supported by grants from the University Nursing Program for Young Scholars with Creative Talents in Heilongjiang Province (UNPYSCT2016193) and Harbin medical university scientific research innovation fund (2017LCZX05) and Fund of scientific research innovation of the First Affiliated Hospital of Harbin Medical University (NO.2018B012).

Availability of data and materials

All data and materials can be provided upon request.

\section{Authors' contributions}

JY performed experiments, analyzed data and wrote the paper; ZHZS, YW and MZ performed some experiments and analyzed data; CS initiated the study, designed experiments and wrote the paper. All authors read and approved the final manuscript.

\section{Ethics approval and consent to participate}

This study was approved by the Ethics Committee of the First Affiliated Hospital of Harbin Medical University. All written informed consents were received from all patients.

\section{Consent for publication}

The authors agree for publication.

\section{Competing interests}

The authors declare that they have no competing interests.

\section{Publisher's Note}

Springer Nature remains neutral with regard to jurisdictional claims in published maps and institutional affiliations.

\section{Author details}

${ }^{1}$ Department of General Surgery, the Sanya Hongsen Hospital of Harbin Medical University, Fenghuang Road, Sanya 572000, China. ${ }^{2}$ Department of General Surgery, the First Affiliated Hospital of Harbin Medical University, Harbin 150001, China. ${ }^{3}$ Department of Pharmacology and Toxicology, Wright State University, Fairborn, $\mathrm{OH}$ 45435, USA. ${ }^{4}$ Department of Ultrasonography, the Sanya Hongsen Hospital of Harbin Medical University, Sanya 572000, China.

Received: 28 June 2018 Accepted: 29 August 2018

Published online: 10 September 2018

References

1. Siegel R, Ma J, Zou Z, Jemal A. Cancer statistics, 2014. CA Cancer J Clin. 2014;64:9-29.

2. Li T, Lai Q, Wang S, Cai J, Xiao Z, Deng D, He L, Jiao H, Ye Y, Liang L, et al. MicroRNA-224 sustains Wnt/beta-catenin signaling and promotes aggressive phenotype of colorectal cancer. J Exp Clin Cancer Res. 2016;35:21.

3. Song $G H$, Xu SF, Zhang $H$, Wang YP, Xiao $C$, Jiang $T$, Wu LL, Zhang $T$, Sun $X$, Zhong $L$, et al. TIMP1 is a prognostic marker for the progression and metastasis of colon cancer through FAK-PI3K/AKT and MAPK pathway. J Exp Clin Cancer Res. 2016;35:148

4. Wei R, Yang Q, Han B, Li Y, Yao K, Yang X, Chen Z, Yang S, Zhou J, Li M, et al. microRNA-375 inhibits colorectal cancer cells proliferation by downregulating JAK2/STAT3 and MAP3K8/ERK signaling pathways. Oncotarget. 2017:8:16633-41.

5. Kapranov P, Cheng J, Dike S, Nix DA, Duttagupta R, Willingham AT, Stadler PF, Hertel J, Hackermuller J, Hofacker IL, et al. RNA maps reveal new RNA classes and a possible function for pervasive transcription. Science. 2007:316:1484-8

6. Ponting $C P$, Oliver PL, Reik W. Evolution and functions of long noncoding RNAs. Cell. 2009:136:629-41.

7. Guttman M, Garber M, Levin JZ, Donaghey J, Robinson J, Adiconis X, Fan L, Koziol MJ, Gnirke A, Nusbaum C, et al. Ab initio reconstruction of cell typespecific transcriptomes in mouse reveals the conserved multi-exonic structure of lincRNAs. Nat Biotechnol. 2010;28:503-10.

8. Klattenhoff CA, Scheuermann JC, Surface LE, Bradley RK, Fields PA Steinhauser ML, Ding H, Butty VL, Torrey L, Haas S, et al. Braveheart, a long noncoding RNA required for cardiovascular lineage commitment. Cell. 2013;152:570-83. 
9. Sigova AA, Mullen AC, Molinie B, Gupta S, Orlando DA, Guenther MG, Almada AE, Lin C, Sharp PA, Giallourakis CC, Young RA. Divergent transcription of long noncoding RNA/mRNA gene pairs in embryonic stem cells. Proc Natl Acad Sci U S A. 2013:110:2876-81.

10. Karlic R, Ganesh S, Franke V, Svobodova E, Urbanova J, Suzuki Y, Aoki F, Vlahovicek K, Svoboda P. Long non-coding RNA exchange during the oocyte-to-embryo transition in mice. DNA Res. 2017;24:129-41.

11. Liu B, Ye B, Yang L, Zhu X, Huang G, Zhu P, Du Y, Wu J, Qin X, Chen R, et al. Long noncoding RNA IncKdm $2 b$ is required for ILC3 maintenance by initiation of Zfp292 expression. Nat Immunol. 2017;18:499-508.

12. Liu J, Liu L, Wan JX, Song Y. Long noncoding RNA SNHG20 promotes gastric cancer progression by inhibiting p21 expression and regulating the GSK-3beta/ beta-catenin signaling pathway. Oncotarget. 2017;8:80700-8.

13. Xu YC, Liang CJ, Zhang DX, Li GQ, Gao X, Fu JZ, Xia F, Ji JJ, Zhang LJ, Li GM, Wu JX. LncSHRG promotes hepatocellular carcinoma progression by activating HES6. Oncotarget. 2017;8:70630-41.

14. Zhu P, Wang Y, Wu J, Huang G, Liu B, Ye B, Du Y, Gao G, Tian Y, He L, Fan Z. LncBRM initiates YAP1 signalling activation to drive self-renewal of liver cancer stem cells. Nat Commun. 2016;7:13608.

15. Xu Y, Leng K, Li Z, Zhang F, Zhong X, Kang P, Jiang X, Cui Y. The prognostic potential and carcinogenesis of long non-coding RNA TUG1 in human cholangiocarcinoma. Oncotarget. 2017;8:65823-35.

16. Zhu J, Shi H, Liu H, Wang X, Li F. Long non-coding RNA TUG1 promotes cervical cancer progression by regulating the miR-138-5p-SIRT1 axis. Oncotarget. 2017;8:65253-64.

17. Ma ZH, Huang HSY, Wang JR, Zhou Y, Pu FX, Zhao QH, Peng P, Hui BQ, Ji H, Wang KM. Long non-coding RNA SNHG15 inhibits P15 and KLF2 expression to promote pancreatic cancer proliferation through EZH2-mediated H3K27me3. Oncotarget. 2017:8:84153-67.

18. Yang J, Li C, Mudd A, Gu X. LnCRNA PVT1 predicts prognosis and regulates tumor growth in prostate cancer. Biosci Biotechnol Biochem. 2017;81(12):2301-6.

19. Wang S, Liang K, Hu Q, Li P, Song J, Yang Y, Yao J, Mangala LS, Li C, Yang W, et al. JAK2-binding long noncoding RNA promotes breast cancer brain metastasis. J Clin Invest. 2017;127(12):4498-515

20. Li J, Xue W, Lv J, Han P, Liu Y, Cui B. Identification of potential long noncoding RNA biomarkers associated with the progression of colon cancer. Oncotarget. 2017:8:75834-43.

21. Yu XF, Mi L, Dong J, Zou J. Long intergenic non-protein-coding RNA 1567 (LINC01567) acts as a "sponge" against microRNA-93 in regulating the proliferation and tumorigenesis of human colon cancer stem cells. BMC Cancer. 2017;17:716

22. Huang JZ, Chen M, Chen D, Gao XC, Zhu S, Huang HY, Hu M, Zhu HF, Yan GR. A peptide encoded by a putative IncRNA HOXB-AS3 suppresses Colon Cancer growth. Mol Cell. 2017;68:171.

23. Shen XG, Bai YF, Luo B, Zhou XG. Upregulation of IncRNA BANCR associated with the lymph node metastasis and poor prognosis in colorectal cancer. Biol Res. 2017;50:32

24. Liu L, Zhang Y, Wong CC, Zhang J, Dong Y, Li X, Kang W, Chan FKL, Sung JJY, YU J. RNF6 promotes colorectal Cancer by activating the Wnt/betacatenin pathway via ubiquitination of TLE3. Cancer Res. 2018;78:1958-71.

25. Liu BY, Ye BQ, Zhu XX, Huang GL, Yang LL, Zhu PP, Du Y, Wu JY, Meng S, Tian Y, Fan ZS. IL-7R alpha glutamylation and activation of transcription factor Sall3 promote group 3 ILC development. Nat Commun. 2017:8:231

26. Zhu P, Wang Y, Huang G, Ye B, Liu B, Wu J, Du Y, He L, Fan Z. Lnc-betaCatm elicits EZH2-dependent beta-catenin stabilization and sustains liver CSC self-renewal. Nat Struct Mol Biol. 2016;23:631-9.

27. Tseng YY, Moriarity BS, Gong W, Akiyama R, Tiwari A, Kawakami H, Ronning P, Reuland B, Guenther K, Beadnell TC, et al. PVT1 dependence in cancer with MYC copy-number increase. Nature. 2014;512:82-6.

28. Yang Q, Wang Y, Pan X, Ye J, Gan S, Qu F, Chen L, Chu C, Gao Y, Cui X. Frizzled 8 promotes the cell proliferation and metastasis of renal cell carcinoma. Oncotarget. 2017;8:78989-9002.

29. Bayin NS, Frenster JD, Sen R, Si S, Modrek AS, Galifianakis N, Dolgalev I, Ortenzi $\mathrm{V}$, Illa-Bochaca I, Khahera A, et al. Notch signaling regulates metabolic heterogeneity in glioblastoma stem cells. Oncotarget. 2017:8:64932-53.

30. Wu G, Xu G, Schulman BA, Jeffrey PD, Harper JW, Pavletich NP. Structure of a beta-TrCP1-Skp1-beta-catenin complex: destruction motif binding and lysine specificity of the SCF(beta-TrCP1) ubiquitin ligase. Mol Cell. 2003;11:1445-56.

31. Wapinski O, Chang HY. Long noncoding RNAs and human disease. Trends Cell Biol. 2011;21:354-61.
32. Majidinia M, Aghazadeh J, Jahanban-Esfahlani R, Yousefi B. The roles of Wnt/ beta-catenin pathway in tissue development and regenerative medicine. J Cell Physiol. 2018;233(8):5598-612.

33. De Boer J, Wang HJ, Van Blitterswijk C. Effects of Wnt signaling on proliferation and differentiation of human mesenchymal stem cells. Tissue Eng. 2004;10:393-401.

34. Yang J, Wei D, Wang W, Shen B, Xu S, Cao Y. TRAF4 enhances oral squamous cell carcinoma cell growth, invasion and migration by Wnt-betacatenin signaling pathway. Int J Clin Exp Pathol. 2015;8:11837-46.

35. Clevers H. Wnt/beta-catenin signaling in development and disease. Cell. 2006;127:469-80

36. White BD, Chien AJ, Dawson DW. Dysregulation of Wnt/beta-catenin signaling in gastrointestinal cancers. Gastroenterology. 2012;142:219-32.

37. Wong CM, Fan ST. Ng IO: beta-catenin mutation and overexpression in hepatocellular carcinoma: clinicopathologic and prognostic significance. Cancer. 2001;92:136-45.

38. Zhang J, Gao YL. CCAT-1 promotes proliferation and inhibits apoptosis of cervical cancer cells via the Wnt signaling pathway. Oncotarget. 2017:8:68059-70.

39. Wang JL, Qi Z, Li YH, Zhao HM, Chen YG, Fu W. TGF beta induced factor homeobox 1 promotes colorectal cancer development through activating Wnt/beta-catenin signaling. Oncotarget. 2017;8:70214-25.

40. Rapicavoli NA, Poth EM, Zhu H, Blackshaw S. The long noncoding RNA Six3OS acts in trans to regulate retinal development by modulating Six3 activity. Neural Dev. 2011;6:32.

41. Yoon JH, Abdelmohsen K, Kim J, Yang X, Martindale JL, Tominaga-Yamanaka K, White EJ, Orjalo AV, Rinn JL, Kreft SG, et al. Scaffold function of long noncoding RNA HOTAIR in protein ubiquitination. Nat Commun. 2013;4:2939.

42. Wang D, Ding L, Wang L, Zhao Y, Sun Z, Karnes RJ, Zhang J, Huang $H$. LncRNA MALAT1 enhances oncogenic activities of EZH2 in castrationresistant prostate cancer. Oncotarget. 2015;6:41045-55.

43. MacDonald BT, Tamai K, He X. Wnt/beta-catenin signaling: components, mechanisms, and diseases. Dev Cell. 2009;17:9-26.

44. Moon RT, Kohn AD, De Ferrari GV, Kaykas A. WNT and beta-catenin signalling: diseases and therapies. Nat Rev Genet. 2004;5:689-99.

45. Liu CM, Li YM, Semenov M, Han C, Baeg GH, Tan Y, Zhang ZH, Lin XH, He X. Control of beta-catenin phosphorylation/degradation by a dual-kinase mechanism. Cell. 2002;108:837-47.

46. Hart M, Concordet JP, Lassot I, Albert I, del los Santos R, Durand H, Perret C, Rubinfeld B, Margottin F, Benarous R, Polakis P. The F-box protein beta-TrCP associates with phosphorylated beta-catenin and regulates its activity in the cell. Curr Biol. 1999;9:207-10.

47. Aberle H, Bauer A, Stappert J, Kispert A. Kemler R: beta-catenin is a target for the ubiquitin-proteasome pathway. EMBO J. 1997;16:3797-804.

48. Fodde R, Edelmann W, Yang K, Vanleeuwen C, Carlson C, Renault B, Breukel C, Alt E, Lipkin M, Khan PM, Kucherlapati R. A targeted chain-termination mutation in the mouse Apc gene results in multiple intestinal tumors. Proc Natl Acad Sci U S A. 1994;91:8969-73.

49. Vogelstein B, Papadopoulos N, Velculescu VE, Zhou S, Diaz LA Jr, Kinzler KW. Cancer genome landscapes. Science. 2013;339:1546-58.

50. Guichard C, Amaddeo G, Imbeaud S, Ladeiro Y, Pelletier L, Maad IB, Calderaro J, Bioulac-Sage P, Letexier M, Degos F, et al. Integrated analysis of somatic mutations and focal copy-number changes identifies key genes and pathways in hepatocellular carcinoma. Nat Genet. 2012;44:694-8.

51. Nakao K, Mehta KR, Fridlyand J, Moore DH, Jain AN, Lafuente A, Wiencke JW, Terdiman JP, Waldman FM. High-resolution analysis of DNA copy number alterations in colorectal cancer by array-based comparative genomic hybridization. Carcinogenesis. 2004;25:1345-57. 